\title{
Martin Kaltenbach
}

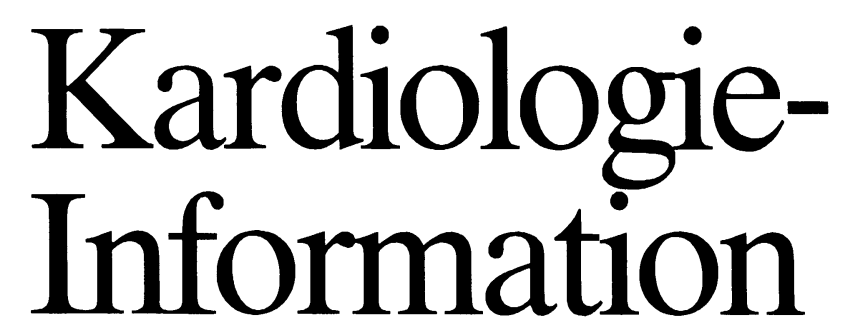

Springer-Verlag Berlin Heidelberg $\mathrm{GmbH}$ 
Prof. Dr. Martin Kaltenbach

Zentrum Innere Medizin

Abteilung Kardiologie

Klinikum der

Johann-Wolfgang Goethe-Universität

Theodor-Stern-Kai 7

6000 Frankfurt 70

CIP-Kurztitelaufnahme der Deutschen Bibliothek

Kaltenbach, Martin:

Kardiologie-Information / Martin Kaltenbach.

ISBN 978-3-7985-0742-5 ISBN 978-3-662-12139-9 (eBook)

DOI 10.1007/978-3-662-12139-9

Dieses Werk ist urheberrechtlich geschützt. Die dadurch begründeten Rechte, insbesondere die der Übersetzung, des Nachdrucks, des Vortrags, der Entnahme von Abbildungen und Tabellen, der Funksendung, der Mikroverfilmung oder der Vervielfältigung auf anderen Wegen und der Speicherung in Datenverarbeitungsanlagen, bleiben, auch bei nur auszugsweiser Verwertung, vorbehalten. Eine Vervielfältigung dieses Werkes oder von Teilen dieses Werkes ist auch im Einzelfall nur in den Grenzen der gesetzlichen Bestimmungen des Urheberrechtsgesetzes der Bundesrepublik Deutschland vom 9. September 1965 in der Fassung vom 24. Juni 1985 zulässig. Sie ist grundsätzlich vergütungspflichtig. Zuwiderhandlungen unterliegen den Strafbestimmungen des Urheberrechtsgesetzes.

Copyright (c) 1988 by Springer-Verlag Berlin Heidelberg

Ursprünglich erschienen bei Dr. Dietrich Steinkopff Verlag, GmbH \& Co. KG, Darmstadt 1988

Verlagsredaktion: Juliane K. Weller - Herstellung: Heinz J. Schäfer

Gesamtherstellung: Druckhaus Beltz, 6944 Hemsbach 


\section{Vorwort}

Während noch vor einigen Dekaden die Innere Medizin als „Lehre von den unheilbaren Krankheiten" galt, sind heute auf vielen Gebieten, ganz besonders dem der HerzKreislauferkrankungen, die therapeutischen Möglichkeiten sehr groß geworden. Allerdings hängt der Einsatz gezielter Therapieverfahren von einer genauen Diagnose ab. Diese kann manchmal nur durch aufwendige diagnostische Eingriffe gestellt werden. Der Einsatz diagnostischer Maßnahmen muß sich nach den zu erwartenden therapeutischen Konsequenzen richten. Um diese abzuschätzen, ist das Verständnis pathophysiologischer Zusammenhänge genauso wichtig wie das systematische Vorgehen, beginnend mit einer individuellen Anamnese.

Das vorliegende Buch ist aus ärztlicher Tätigkeit und aus mehr als 30jähriger Vorlesungs- und Fortbildungserfahrung in der Überzeugung entstanden, daß die moderne Kardiologie ein Gebiet ist, das weit mehr durch Erkennen logischer Zusammenhänge als durch Vermehrung von Wissensstoff erarbeitet werden muß.

Die Einteilung des Buches folgt praktischen Gesichtspunkten und orientiert sich an den einzelnen Krankheitsbildern entsprechend ihrer Häufigkeit und sozialmedizinischen Bedeutung weit mehr als am Gegenstandskatalog. Es soll dem Studenten oder dem Arzt das Verständnis von Herz-Kreislauferkrankungen erleichtern. Jede Krankheit ist ein dynamischer Prozeß, der in Verbindung mit dem gesamten Organismus und der Persönlichkeit des Kranken gesehen und beurteilt werden muß. Dies hat der Spezialist und der Allgemeinarzt in gleicher Weise zu berücksichtigen.

Viele Anregungen habe ich aus Kreisen der Studenten, Doktoranden und der Ärzteschaft empfangen. Zu danken habe ich meinen Mitarbeitern, in erster Linie Herrn Wolfgang Schneider und Frau Elisabeth Neizert. Besonders dankbar bin ich auch meinem Lehrer Helmut Klepzig, den Kollegen der Herzchirurgie und Anästhesiologie sowie der Radiologie, Pathologie, Physiologie und Nuklearmedizin für die langjährige und fruchtbare Zusammenarbeit und die Überlassung von Befunden.

Das Buch möge das Verständnis für Herz-Kreislaufkranke fördern und helfen, moderne Medizin menschlich zu praktizieren. Das kann nur gelingen, wenn der Arzt den Stellenwert diagnostischer und therapeutischer Maßnahmen kennt und versteht. Nur dann wird es ihm gelingen, für jeden einzelnen Kranken den richtigen Weg zwischen dem medizinisch Möglichen und dem menschlich Angemessenen zu finden. 


\section{Inhaltsverzeichnis}

Vorwort

1 Bedeutung der Herz-Kreislauferkrankungen für Morbidität und Mortalität ....................... 1

2 Erhebung der Anamnese und körperliche Untersuchung . . . . . . 3

$2.1 \quad$ Anamnese . . . . . . . . . . . . . . . . 3

2.2 Körperliche Untersuchung . . . . . . . . . . . . . 5

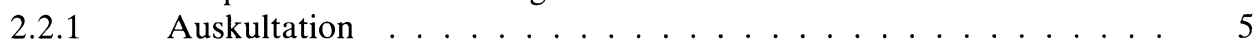

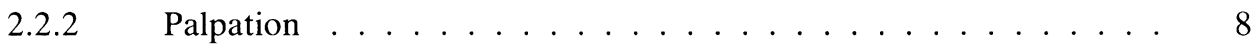

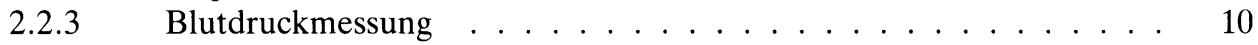

2.2.4 Dokumentation ...................... 11

3 Herz-Kreislaufuntersuchungen (außer Ergometrie, s. 4.2.2) . . . . 13

$3.1 \quad$ Elektrokardiographie . . . . . . . . . . . . . . 13

3.2 Phonokardiographie, Pulskurven ............. 20

3.3 Echokardiographie ................. 21

3.4 Indikatorverdünnungsmethode . . . . . . . . . . . 23

3.5 Röntgenuntersuchung des Thorax und Herzvolumenbestimmung . . . 25

$3.6 \quad$ Nuklearmedizinische Verfahren (siehe auch 4.2.3) . . . . . . . . . . 29

3.6.1 Radionuklidventrikulographie . . . . . . . . . . . . 29

3.6.2 Thalliumszintigraphie . . . . . . . . . . . . . . 30

3.7 Computertomographie, Positronenemissionskamera, Kernspintomo-

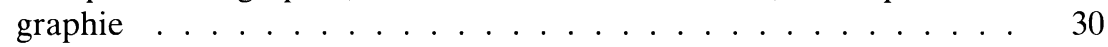

$3.8 \quad$ Herzkatheterismus . . . . . . . . . . . . 30

$4 \quad$ Koronare Herzerkrankung . . . . . . . . . . . . . 33

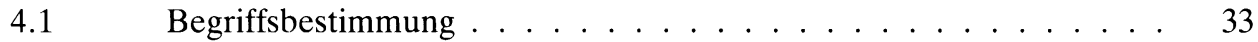

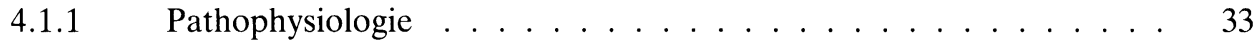

4.1 .2 Atheromatose . . . . . . . . . . . . . . 34

4.1.3 Herzmuskeldurchblutung, Kranzarteriensystem . . . . . . . . 35

4.1.4 Kollateralen . . . . . . . . . . . . . . . . . 38

4.1.5 Entwicklung der stenosierenden Koronarsklerose . . . . . . . . . . . 39

4.1.6 Bedeutung der koronaren Herzerkrankung, Risikofaktoren . . . . . . 39

4.1 .7 Prognose . . . . . . . . . . . . . . . . . 44

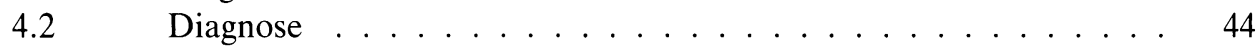

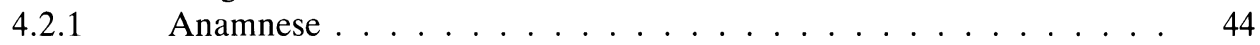

4.2.2 Ergometrie, Belastungs-EKG . . . . . . . . . . . . . 46

4.2.3 Nuklearmedizinische Verfahren (siehe auch 3.6) . . . . . . . . 52 
$4.2 .4 \quad$ Koronarangiographie . . . . . . . . . . . . . . . . 53

$4.3 \quad$ Verlaufsformen der Angina pectoris . . . . . . . . . . . 55

4.3.1 Stabile Angina pectoris . . . . . . . . . . . . . . . 55

4.3.2 Instabile Angina pectoris _ . . . . . . . . . . . . . . . . 55

4.3.3 Ruhe-Angina-pectoris . . . . . . . . . . . . . . 56

4.4 Differentialdiagnose von Angina pectoris, kardialen und extrakardialen Brustschmerzen . . . . . . . . . . . . . . . . . 56

4.5 Therapie der Angina pectoris $\ldots \ldots \ldots \ldots$

4.5.1 Allgemeinmaßnahmen . . . . . . . . . . . . . . . 59

4.5.2 Antianginöse Medikamente, Thromboseverhütung . . . . . . . . 60

4.5.3 Wirkungsmechanismus antianginöser Medikamente . . . . . . . 61

4.5.4 Revaskularisation durch Operation und Ballondilatation . . . . . . 62

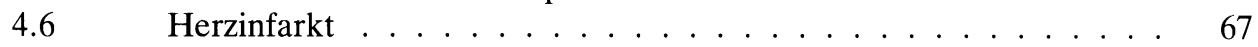

4.6 .1 Definition, Einteilung . . . . . . . . . . . . . . . 67

4.6.2 Infarktentstehung . . . . . . . . . . . . . . . 68

4.6.3 Pathologisch-anatomische, angiographische und angioskopische Befunde . . . . . . . . . . . . . . . . . . . 68

4.6.4 Klinisches Bild . . . . . . . . . . . . . . . . . . . . . . . . . 69

4.6 .5 Diagnose . . . . . . . . . . . . . . . . 70

4.6.6 Therapeutische Maßnahmen und diagnostische Schritte beim frischen Herzinfarkt . . . . . . . . . . . . . . . . . 71

4.6.7 Der komplizierte Myokardinfarkt, Herzwandaneurysma _ . . . . . 75

4.6.8 Mobilisierung, diagnostische Maßnahmen nach Infarkt . . . . . . 76

$4.6 .9 \quad$ Nichttransmuraler Infarkt . . . . . . . . . . . . . . . . . 78

4.6.10 Rehabilitation . . . . . . . . . . . . . . . . . . . 79

4.6.11 Psychosomatische Behandlung des Infarktpatienten . . . . . . . . . 79

4.6.12 Allgemeine Lebensweise, medikamentöse Dauerbehandlung . . . . 80

$5 \quad$ Entzündliche Herzerkrankungen $\ldots \ldots \ldots \ldots \ldots$

$5.1 \quad$ Endokarditis . . . . . . . . . . . . . . . . 81

5.1.1 Bakterielle Endokarditis . . . . . . . . . . . . 81

5.1.2 Rheumatische Endokarditis . . . . . . . . . . . . . . . 83

5.1.3 Seltene Endokarditisformen $\ldots \ldots \ldots \ldots$. . . . . . . . . 84

$5.2 \quad$ Myokarditis, Perikarditis . . . . . . . . . . . . 84

$6 \quad$ Aortenerkrankungen . . . . . . . . . . . . 88

6.1 Entzündliche Erkrankungen der Aorta, luetische und Takayasu-Aorti-

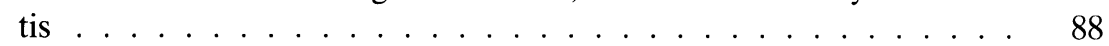

$6.2 \quad$ Aortenaneurysmen $\ldots \ldots \ldots \ldots \ldots$

$7 \quad$ Herzklappenfehler . . . . . . . . . . . . . . . 90

$7.1 \quad$ Bedeutung, Einteilung, Entstehung . . . . . . . . . . . 90

$7.2 \quad$ Mitralstenose . . . . . . . . . . . . . . . . . . . . 91

$7.2 .1 \quad$ Entstehung . . . . . . . . . . . . . . . . . . . . 91

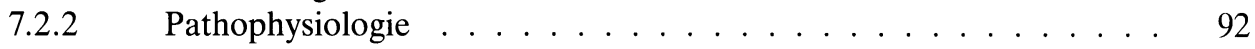

$7.2 .3 \quad$ Klinik und Verlauf . . . . . . . . . . . . . . . . . . . . . . . . 92

7.2 .4 Therapie .................... 96 
$7.3 \quad$ Mitralinsuffizienz . . . . . . . . . . . . . . 96

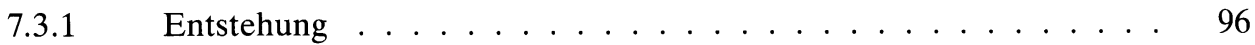

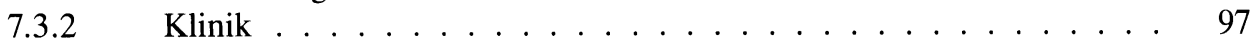

7.3.3 Verlauf, Therapie . . . . . . . . . . . . . . . . 98

7.3.4 Antikoagulation bei Mitralvitien . . . . . . . . . . . . . 98

$7.4 \quad$ Aortenstenose . . . . . . . . . . . . . . . . . 98

7.4.1 Vorkommen, Entstehung . . . . . . . . . . . . 98

7.4.2 Klinik . . . . . . . . . . . . . . . . . . . . . . . . 99

7.4.3 Verlauf, Therapie . . . . . . . . . . . . . . . 100

7.4.4 Operationsindikation und -verfahren . . . . . . . . . . . . . . . 100

7.5 Aorteninsuffizienz . . . . . . . . . . . . . . 101

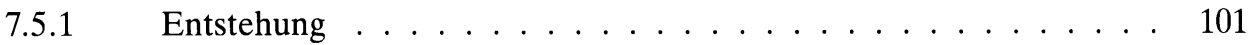

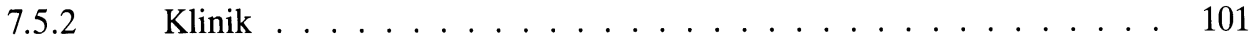

7.5.3 Verlauf, Therapie . . . . . . . . . . . . . . . 103

7.6 Pulmonalklappenfehler . . . . . . . . . . . . . . . 104

7.6.1 Vorkommen ......................... 104

7.6.2 Pathologie, Pathophysiologie . . . . . . . . . . . . . . 104

7.6.3 Klinik . . . . . . . . . . . . . . . . . . 104

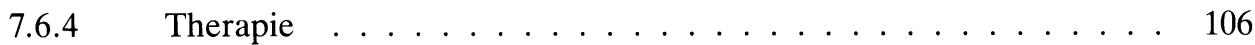

7.7 Trikuspidalstenose . . . . . . . . . . . . . . 106

7.7.1 Pathologie, Pathophysiologie, Vorkommen . . . . . . . . . . 106

7.7.2 Klinik, Therapie . . . . . . . . . . . . . . . . 107

7.8 Trikuspidalinsuffizienz . . . . . . . . . . . . . . . 107

7.8.1 Vorkommen, Entstehung . . . . . . . . . . . . . 107

7.8.2 Klinik, Therapie . . . . . . . . . . . . . . . 107

$8 \quad$ Mißbildungen des Herzens und der großen Gefäße ～. . . . . . . . . . 109

$8.1 \quad$ Vorhofseptumdefekt . . . . . . . . . . . . . . . 109

8.1.1 Pathologie, Pathophysiologie, Vorkommen . . . . . . . . . . . . . 109

8.1.2 Klinik, Verlauf, Therapie . . . . . . . . . . . . . . . . . . 110

8.2 Ventrikelseptumdefekt . . . . . . . . . . . . . . . . 114

8.2.1 Pathologie, Pathophysiologie, Vorkommen . . . . . . . . . . . . . 114

8.2.2 Verlauf, Klinik, Therapie . . . . . . . . . . . . . . . 115

8.3 Aortenisthmusstenose . . . . . . . . . . . . . . 116

8.3.1 Pathologie . . . . . . . . . . . . . . . 116

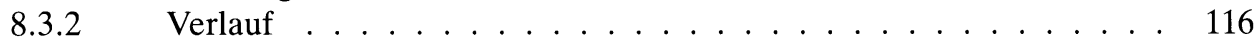

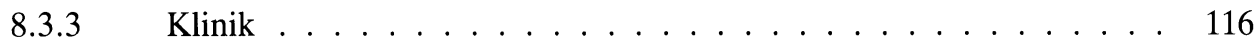

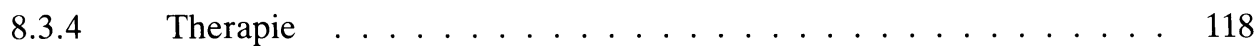

$8.4 \quad$ Persistierender Ductus Botalli . . . . . . . . . . . . . . 118

8.4.1 Vorkommen, Pathologie . . . . . . . . . . . 118

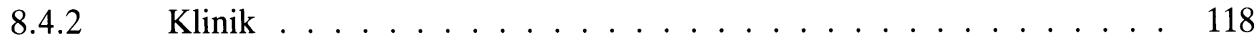

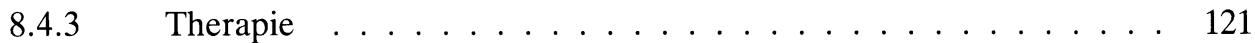

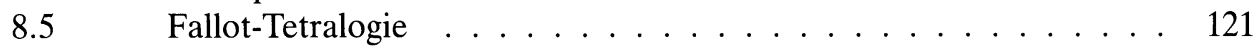

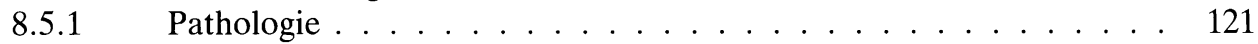

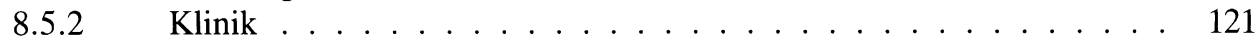

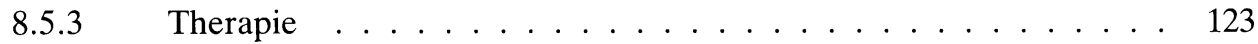

8.6 Transposition der großen Gefäße . . . . . . . . . . . . . . . 123 
9 Herzmuskelerkrankungen . . . . . . . . . . . . . . . . 124

9.1 Begriffsbestimmung, Entstehung, Einteilung . . . . . . . . . . . 124

9.1.1 Pathologie, Pathogenese, Pathophysiologie . . . . . . . . . . . . 124

9.2 Dilative Myokardiopathie . . . . . . . . . . . . . . . . . . . 125

9.2.1 Definition, Vorkommen . . . . . . . . . . . . . . . 125

9.2.2 Pathologie, Pathogenese, Pathophysiologie . . . . . . . . . . 126

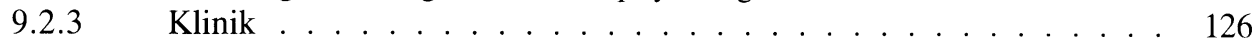

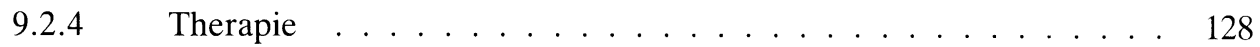

9.3 Hypertrophische Myokardiopathie . . . . . . . . . . . . 130

9.3.1 Definition .......................... 130

9.3.2 Vorkommen, Pathologie, Pathogenese . . . . . . . . . . . 130

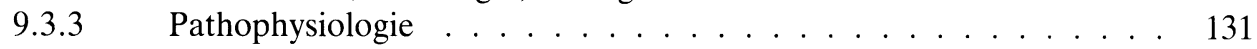

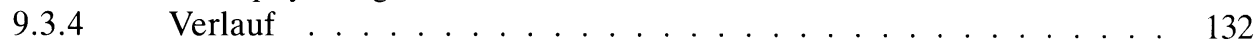

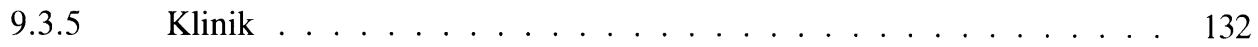

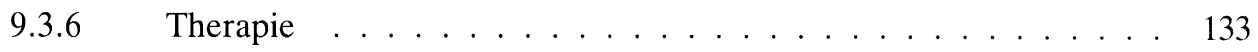

9.4 Restriktive Myokardiopathien . . . . . . . . . . . . 135

$10 \quad$ Hypertonie im großen und kleinen Kreislauf . . . . . . . . . . . . . . 136

10.1 Hypertonie im großen Kreislauf . . . . . . . . . . . . . . . . . . . . 136

10.1.1 Definition des erhöhten Blutdrucks . . . . . . . . . . . . . . 136

10.1.2 Pathogenese . . . . . . . . . . . . . . 136

10.1.3 Formen der Blutdruckerhöhung . . . . . . . . . . . . . . . . . 137

10.1.4 Klinik . . . . . . . . . . . . . . . . . . . . 137

10.1.5 Behandlungsprinzipien, Allgemeinmaßnahmen . . . . . . . . . . 137

10.1.5.1 Medikamente . . . . . . . . . . . . . . . . . . . 138

10.1.5.1.1 Kalziumantagonisten . . . . . . . . . . . . . . . . . . 138

10.1.5.1.2 Betarezeptorenblocker . . . . . . . . . . . . . . . . . . 138

10.1.5.1.3 Alphablocker, periphere Vasodilatantien . . . . . . . . . . . . 138

10.1.5.1.4 Clonidin und Metyldopa . . . . . . . . . . . . . . . . . . . . . . . . 139

10.1.5.1.5 Angiotensin-Converting-Enzym(ACE)-Hemmer . . . . . . . . . . 139

10.1.5.1.6 Saluretika . . . . . . . . . . . . . . . . . . . . . 139

10.1.5.2 Behandlung der Blutdruckkrise . . . . . . . . . . . . . . . . . . . . 139

$10.2 \quad$ Hypertonie im kleinen Kreislauf . . . . . . . . . . . . . . . . . . . . 139

10.2.1 Entstehung, Verlauf . . . . . . . . . . . . . . . . . . . 139

10.2.2 Klinik . . . . . . . . . . . . . . . . . . . . . . . . . . . . . . . 140

10.2.3 Therapie ......................... 140

11 Kreislaufregulationsstörungen . . . . . . . . . . . . . 141

$11.1 \quad$ Hyperkinetische und hypertone Regulationsstörungen . . . . . . . . 141

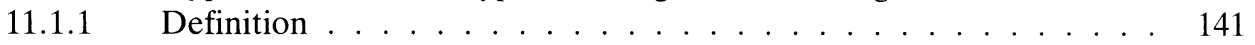

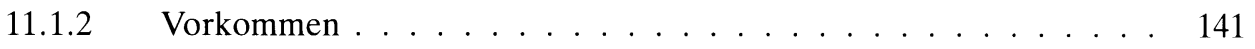

11.1.3 Pathogenese . . . . . . . . . . . . . . . . 141

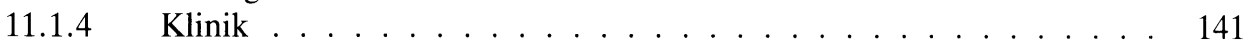

11.1.5 Differentialdiagnose, Verlaufsformen . . . . . . . . . . . . . 144

11.1.6 Therapie ....................... . . . 144 
11.2 Hypodyname und hypotone Kreislaufregulationsstörungen . . . . . . 144

11.2.1 Definition . . . . . . . . . . . . . . . . 144

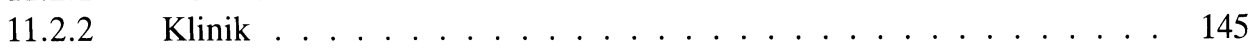

11.2.3 Therapie .................................. 145

11.3 Nervöses Atmungssyndrom . . . . . . . . . . . . . . . . . . . 146

12 Herzrhythmusstörungen . . . . . . . . . . . . . . 147

$12.1 \quad$ Einteilung . . . . . . . . . . . . . . . . 147

12.1.1 Einteilung nach der Grundkrankheit . . . . . . . . . . . . . . 147

12.1.2 Einteilung nach der Herzfrequenz . . . . . . . . . . . . . . . . . . 148

12.1.3 Einteilung nach dem Auftreten . . . . . . . . . . . . . . . . 148

12.1.4 Einteilung nach der Behandlungsbedürftigkeit . . . . . . . . . . . . 148

12.2 Untersuchungsverfahren . . . . . . . . . . . . . . . . 149

12.2.1 Elektrokardiographie . . . . . . . . . . . . . . . . . 149

12.2.2 Langzeitelektrokardiographie . . . . . . . . . . . . . . 149

12.2.3 Ergometrie . . . . . . . . . . . . . . . . . . 149

12.2.4 His-Bündelelektrokardiographie . . . . . . . . . . . . . . 149

12.2.5 Sinusknotenerholungszeit . . . . . . . . . . . . . . 150

12.2.6 Programmierte Stimulation . . . . . . . . . . . . . . . . . 150

12.2.7 Intrakardiales Mapping . . . . . . . . . . . . . . . . . 150

12.3 Ventrikuläre Extrasystolen, ventrikuläre Tachykardie . . . . . . . . 150

12.3.1 Klinik . . . . . . . . . . . . . . . . . 150

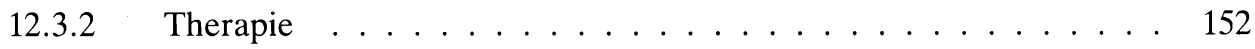

12.4 Supraventrikuläre Extrasystolen, Vorhofflimmern und -flattern, Vorhoftachykardie, paroxysmale supraventrikuläre Tachykardie . . . . . 153

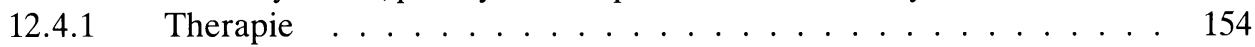

12.5 Bradykarde Rhythmusstörungen . . . . . . . . . . . . 156

12.5.1 Definition, Vorkommen, Klinik . . . . . . . . . . . . . . . 156

12.5.2 Therapie . . . . . . . . . . . . . . . . 158

12.6 Störungen der intraventrikulären Erregungsleitung . . . . . . . . . . 159

12.7 WPW-, LGL- und QT-Syndrom . . . . . . . . . . . . . . . . . 161

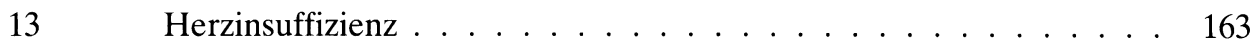

13.1 Definition, Einteilung . . . . . . . . . . . . 163

$13.2 \quad$ Klinik . . . . . . . . . . . . . . . . . 164

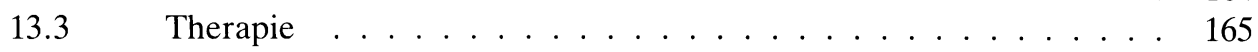

14 Herz-Kreislauferkrankungen und Sport . . . . . . . . . . . . . . . . 167

14.1 Beziehungen zwischen Bau und Funktion des Herzens, Anpassung an vermehrte Belastung . . . . . . . . . . . . . . 167

14.2 Messung der körperlichen Leistungsfähigkeit, Belastungsarten und Meßziele ....................... . . 168

14.3 Gefährdung durch Sport . . . . . . . . . . . . . . . . . . . . . 169

14.4 Sport für den Gesunden . . . . . . . . . . . . . . . . . . . . . . . . 169

14.5 Bewegungstherapie, Sport und Arteriosklerose . . . . . . . . . . . . 170

15 Weiterführende Literatur . . . . . . . . . . . . . . 171

16 Stichwortverzeichnis ..................... 173 